\title{
The spatial controlling of Lamb waves excited by a point source on the cylindrical wall
}

\author{
V. Sukackas \\ Physics Department, Kaunas University of Technology, Lithuania
}

\begin{abstract}
A Lamb wave excited on one point of a hollow cylindrical point reaches every other point in different ways, such as: by the shortest way and turning around 1, $2,3 \ldots$ times. This factor can be used to control the wave field in the space, as it is impossible in the other ways when the source is a point. The waves can be focused if the emitter is excited by a series of short pulses calculated so that at the same time the pulse that has spun the cylinder $n$ times is received, the second pulse with n-1 rotation around the cylinder and so on, and finally the last pulse that came by the shortest way. The focus point can be changed, i.e. scanned when the pulse's position in the series is changed. The only one transducer becomes equivalent to the array that has the period equal to the perimeter of the cylinder. In the same way the signal in the receiving point can be processed by delaying and summing it so that constructive superposition occurred only when the signal comes from the desirable point. In this way the dynamic focusing and scanning can be performed irradiating the object or receiving the waves propagated by some point. In the latter case this can be the point of the passive reflecting defect in pulse-echo NDT mode or the source of the acoustic emission. The scanner is very simple - it consists of one point-form transducer. In the same way the more complicated and typical only to cylinder modes can be excited. Keywords: Lamb waves, cylinder, scanner, dynamic focusing, virtual array.
\end{abstract}

\section{Introduction}

Lamb waves with the propagating medium restricted by two surfaces perfectly suit for the testing of hollow cylinder type objects, such as pipes (Alleyne et $a l$ [1] and Alleyne and Cawley [2]) and their inner surface (Volkovas and Sukackas [3]). Three exciting modes can be distinguished there: 
- The excited signal is close to the continuous waves, i.e. wave burst length is much more longer than the time of passing by the perimeter,

- the burst length is close to the time of passing the perimeter,

- the excited signal is much more shorter.

In the first case the wave components with the wave vector perpendicular to the axis of the cylinder makes standing waves. The cylinder becomes a ring resonator with the parameters carrying information (e.g. about the sediments on cylinder pipe of the inner surface). Resonance signal as a rule is distorted. This reason stimulated the use of untypical solutions for the resonance measurement technique, such as correlation analysis (Sukackas et al [4, 5]).

Sukatskas and Volkovas [6] found that the transmitter and receiver must not necessary be fixed on the same cut (one against the other) and this broadens the possibility of the application.

The formation of the received signal was observed in the second case by Sukackas and Ramanauskas [7]. In this case the use of two - dimensional Fourier transform method (2D FFT) is promising (Alleyne and Cawley [8]). The 2D FFT of the received signal as a function of two variables - carrier frequency and time - allows solving about the attenuation of the waves and about the thickness of the sediment layer inside. The measurement process is much faster as in resonance mode (Sukatskas and Volkovas [9]).

In both cases the integral value of the researched parameter is obtained in all the cut of cylinder. The case with the considerably short excited signal is not enough researched. It has advantages when the local non-homogeneity is researched and allows scanning them with the wave beam.

\section{The principle of controlling}

The layout of transducers on the surface of the pipe is shown in Fig. 1(a), evolvent of waves' path in Fig. 1(b). Using waves that have spun several times, the transmitter becomes equivalent to the virtual phased array with the period $2 \pi R$, where $R$ is the radius of the cylinder.

\subsection{The research of beam focusing}

Both $h_{1}$ and $h_{2}$ in this case are so big that wave reflection from the end of the cylinder is not felt. The transmitter 1 is excited by the pulse burst, the transducer 2 is used for the wave receiving. The time of transmitting of the pulses is chosen so that at the some time the receiver 2 is reached by the latest pulse direct, and by the earlier transmitted - with $1,2, \ldots i$ rotations. In such way we can get the constructive interference.

The passed way of every wave $l_{i}$ is calculated accordingly

and delay time $\tau_{i}$

$$
l_{i}^{2}=h^{2}+\left(a^{2}+2 \pi R(i-1)\right)^{2},
$$

$$
\tau_{i}=l_{i} / c,
$$


where $h$ and $a$-coordinates of the receiving point, $c$ - the velocity of waves, $R$ - radius of the cylinder, $i$ - pulse number ( 1 - the latest).

The example of the excited and received signals is shown in fig. 2 . The steel pipe with $2 R=150 \mathrm{~mm}$ and wall thickness of $8 \mathrm{~mm}$ was analyzed. It was excited with the rectangular pulses of $5 \mu$ s length, that corresponds a half of the period of transducers resonance frequency, $i=1 \ldots 4$.

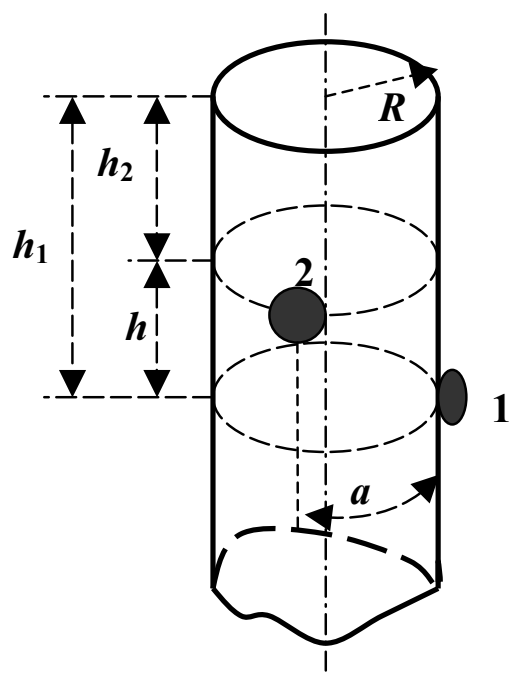

(a)

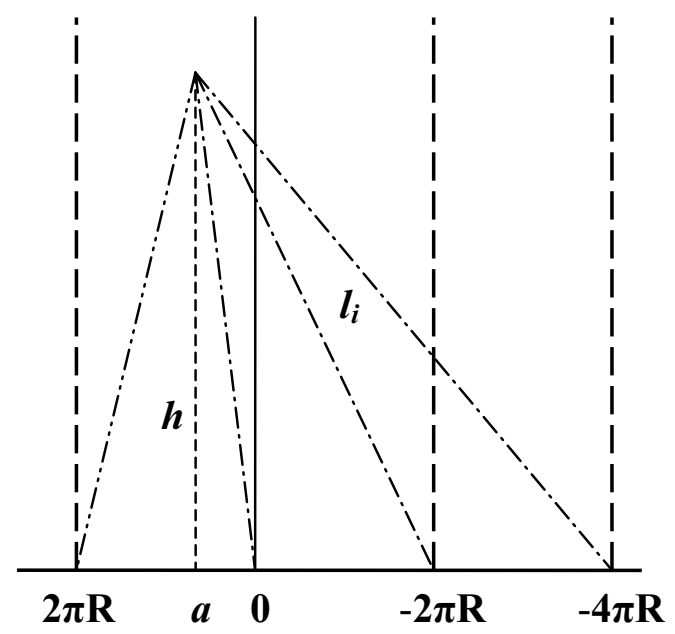

(b)

Figure 1: $\quad$ Layout of the transducers (a), evolvent of waves' path (b). 
The coordinates of the receivers are: $h=400 \mathrm{~mm}, a=240 \mathrm{~mm}=\pi R$; so the images for the waves propagating clockwise and anticlockwise coincide. The sharp increase of the amplitude in the receiving point is observed.

The traditional way for the analysis of directional diagram - to change the position of the receiver - was not used as the acoustic contact stability could not be ensured. It was evaluated by scanning, i.e. changing the focus point calculated according the eqns (1), (2), when the position of the receiver is fixed. The step of scanning on both coordinates is $10 \mathrm{~mm}$. The example to the case with the coordinates of the receiver $\mathrm{h}=400 \mathrm{~mm}, \mathrm{a}=240 \mathrm{~mm}$ is shown in fig.3).

Fig. 3 and the corresponding theoretical research show that directional diagram is oriented to the "center of gravity" of the virtual array. Its position depends also on the damping of the waves.

\subsection{Focusing in the receiving mode}

In this case the transmitter radiates waves once. The receiver registers the direct signal, stores it, then stores the signal that spin once around the cylinder, etc. The appearance time of the signals that must be stored is determined by eqns (1) and (2). After that they are summarized.

The source of the signal can be chosen in the experiment - the transmitter (imitating acoustic emission) or very well reflected defect. The medium version was the imaginary source - reflection of the transmitter 1 from the end of the pipe.

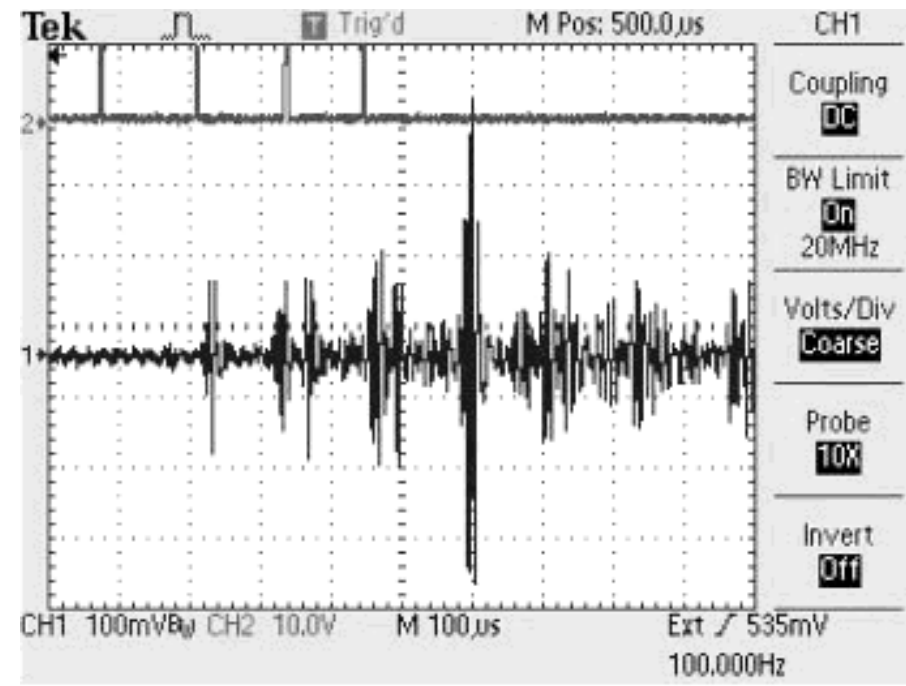

Figure 2: $\quad$ Exciting burst (on the top) and received signal (at the bottom). 


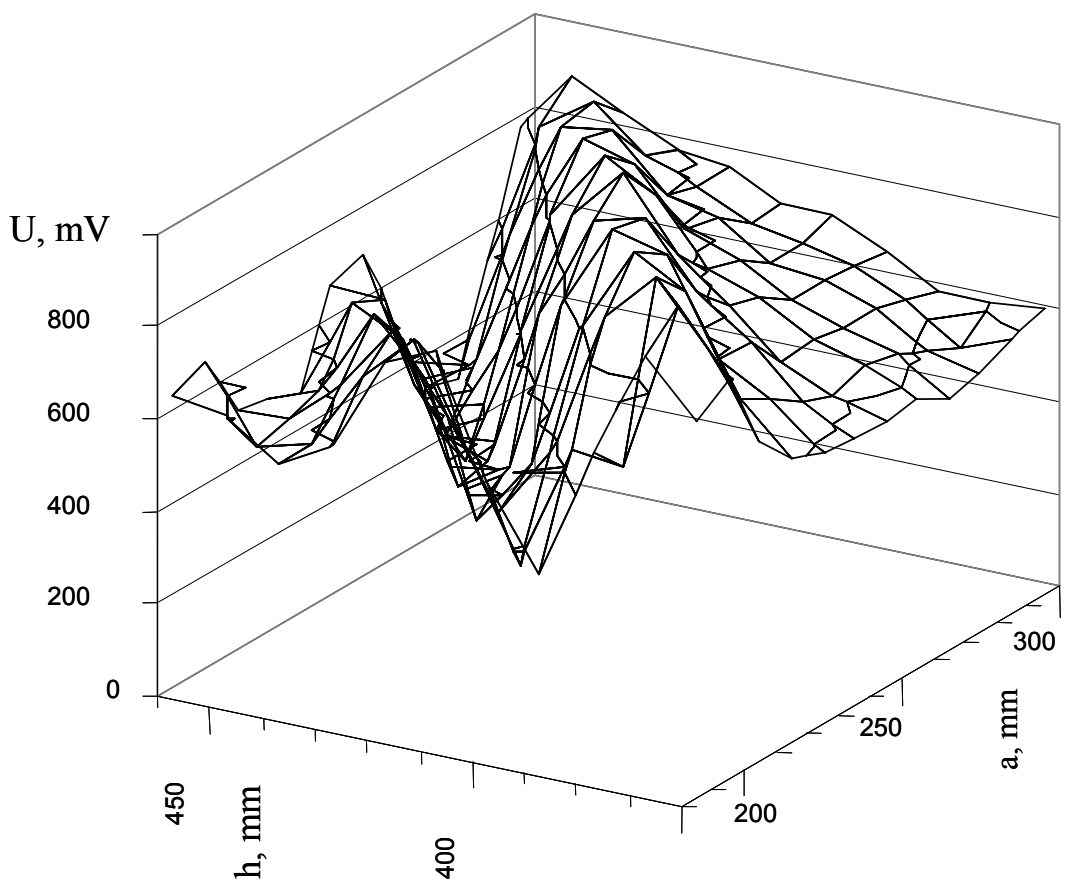

Figure 3: The received signal "peak-peak" obtained by scanning in the rectangular $(190,360)-(190,460)-(300,460)-(300,360)$. The coordinates of the receiver $(240,400)$; the first number is $a$.

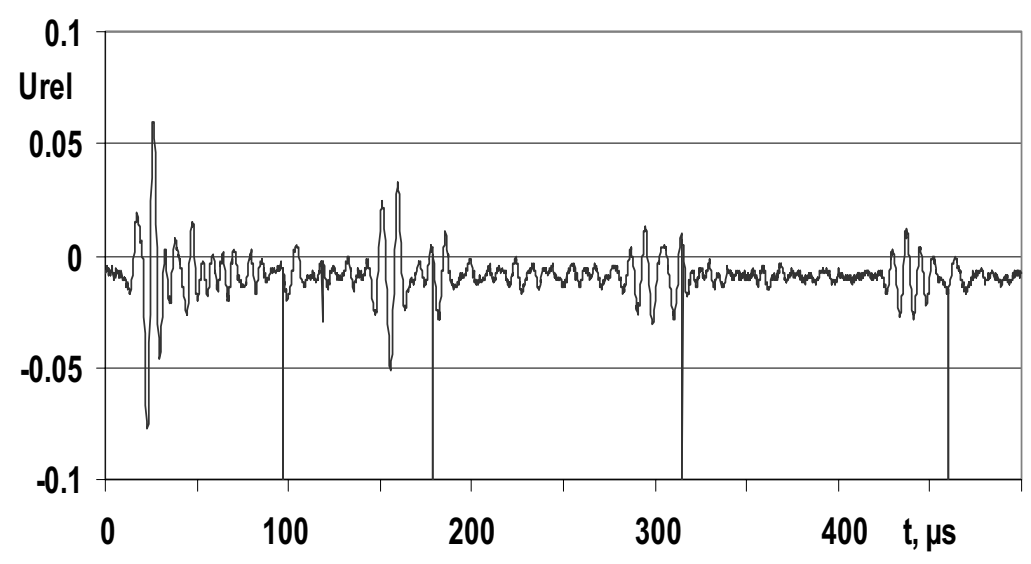

Figure 4: The received signal, when $a=0, h_{1}=150 \mathrm{~mm}, h_{2}=130 \mathrm{~mm}$. The calculated appearance time of the signals from the imaginary source is marked by vertical lines. 


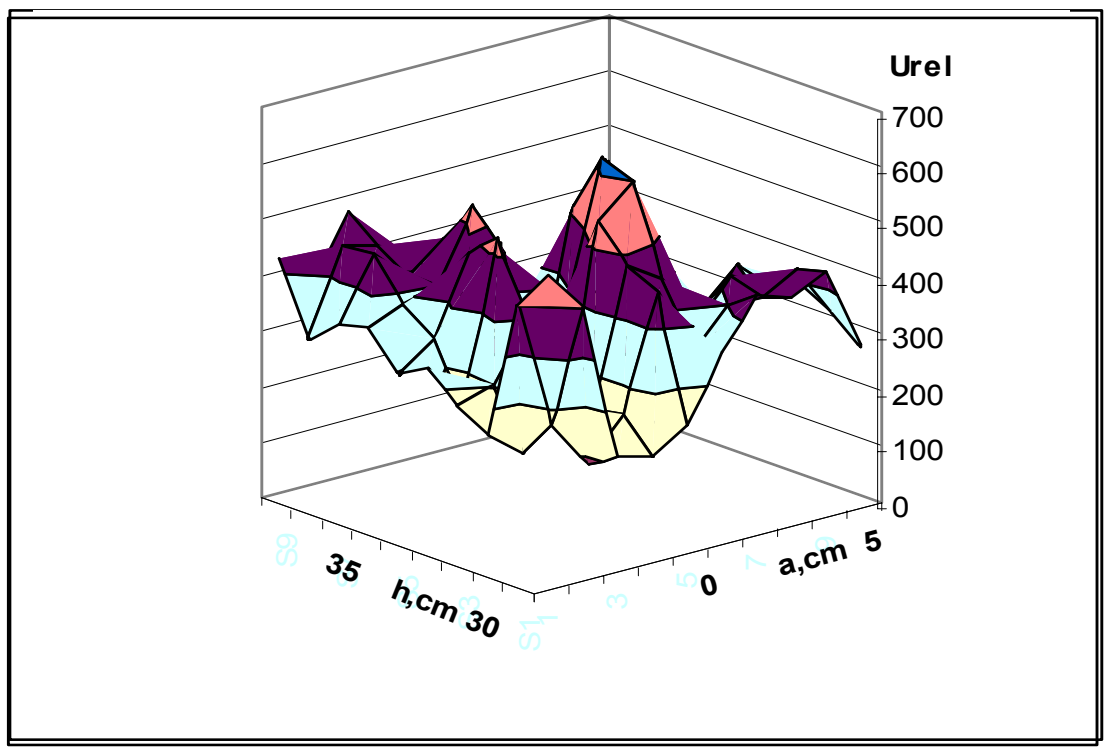

Figure 5: The received signal "peak-peak" obtained by scanning in the rectangular $(-40,290)-(-40,380)-(50,380)-(50,360)$. The first number is $a$.

In this case $a=0, h_{1}=150 \mathrm{~mm}, h_{2}=130 \mathrm{~mm}$ were chosen. The receiver 2 was focused to the point that is at the distance $h_{l}$ above the pipe end, i.e. the distance $h$ in the eqn (1) is chosen to be equal to $h_{1}+h_{2}$. The example of the received signal is shown in Fig. 4. The bigger signals came from the transmitter directly and with 1-3 turns around the cylinder. The time moments that are expected to have pulses from imaginary source (also directly and with 1-3 turns) are marked by vertical lines. At those moments enough intensive signals are really received. The last two pulses as from the transmitter as from the imaginary source almost coincide. So only two first pulses were used for focusing.

The received signal was sampled and stored. The intervals from its appropriate places were selected a bit longer that the transducer own oscillation period $(10 \mu s)$ and the signals were summarized. Such operations are accomplished for the coordinates $\mathrm{a}=-40 \ldots+50 \mathrm{~mm}$ and $\mathrm{h}=290 \ldots 380 \mathrm{~mm}$.

The results of this scanning (voltage "peak-peak") are shown in fig. 5. The imaginary source (the point with coordinates $\mathrm{a}=10, \mathrm{~h}=330 \mathrm{~mm}$ ) is clearly seen. The distance a must be 0 and $\mathrm{h}=280 \mathrm{~mm}$ when the cut at the end of the pipe is flat and perpendicular to the axis; in our case the cut was not such.

\subsection{The effectiveness of focusing}

The intensity of the waves radiated by every array element decreases by their propagating because of two reasons:

- wave scattering,

- damping. 
Those factors influence all the elements almost equally in traditional array constructions, as array period is less that the distance to the focus point. In our case the period $2 \pi R$ is of the same order or longer that the distance $l_{i}($ fig. $1 \mathrm{~b}$ ) and the influence of those factors is different to every wave. Overall, the amplitude $\mathrm{U}$ of the wave in the focus point is equal to the sum of separate wave amplitudes and for the plane wave perpendicular to the cylinder axis, we will have such expression:

$$
U=U_{I}\left(1+r+r^{2} \ldots+r^{i-1}+\ldots\right),
$$

where $U_{I}$ is the amplitude of the first wave, $r=\exp (-2 \pi R \alpha), \alpha$ is the coefficient of the attenuation, $i-$ is the number of the wave. The limit of the eqn (3) when $i$ is infinite:

$$
U=U_{l} /(1-r) .
$$

Because of the scattering every member of the series eqn (3) must be multiplied from the accordingly the member of that series

$$
1+\frac{1}{\sqrt{2}}+\frac{1}{\sqrt{3}}+\ldots \frac{1}{\sqrt{i-1}}+\ldots
$$

It can be seen that according to the case of classical array $U / U_{I}$ is close to the used wave number $n . U / U_{l}<n$ in the analyzed case. Analysis fits as in the mode of transmission as receiving.

\section{Conclusions}

The researched case of the wave control in the space is distinct by the construction that cannot be simplified, as the array consists of the only one alldirectional transducer. But the combinations of $a$ and $h$ must be avoided when not all the waves can coincide. Those cases generate the disturbing noise. Two means can be proposed:

- to use the wave pulses as short as possible,

- not to use the method for the part of the cylinder where it is impossible the coincidence of all waves.

\section{References}

[1] Alleyne, D.N., Lank, A.M., Mudge, P.J. \& Cawley. P., The Lamb wave inspection of chemical plant pipework. Trend in NDE Science \& Technology, Vol. 4, Ashgate publishing company: New Delhi, pp. $2303-$ 2306, 1996.

[2] Alleyne, D.N., .\& Cawley. P., The Interaction of Lamb waves with Defects. IEEE Trans. on Ultr., Ferroel. and Freq. Contr. 39(3), pp. 381- 397, 1992. 
[3] Volkovas, V. \& Sukackas. V., Technical diagnostics of pipe-lines by the approach of wave interference. Proc. of IMEKO XIV World Congress, ed. J. Halttunen, Finnish Soc. of Automation, Tampere, VII, pp. 67-70, 1997.

[4] Sukackas, V., Giedraitienè, V.\& Ramanauskas, R., The non-destructive method of estimation of sediment layer thickness in pipes: problems, algorithms, PC-simulation. Proc. of the $2^{\text {nd }}$ Int. conf. on emerging technologies in NDT, eds. D. Van Hemelrijck, A. Anastassopoulos \& Th. Phillipidis, A. A. Balkema: Rotterdam, pp. 351-356, 2000.

[5] Sukackas, V., Giedraitienè, V.\& Ramanauskas, R., Nondestructive testing of the pipe inner cavity. Proc. of IMEKO XVI World Congress, eds. M.N. Durakbasa, P.H. Osanna \& A. Afjehi-Sadat, Austrian Soc. for Measur. and Autom.: Vienna,VI, pp. 273-277, 2000.

[6] Sukackas, V. \& Volkovas, V., Investigation of Lamb Wave Interference in a Pipeline with Sediments on the Inner Surface. Russ. Journ. of NDT., 39(6), pp. $445-453,2003$.

[7] Sukackas, V.\& Ramanauskas, R., A PC Simulation of the Propagation of the Lamb Waves in Pipes. Proc. of the $3^{\text {nd }}$ Int. conf. "Acoustics III", eds. D. Almorza, C. A. Brebbia, R. Hernandez, WIT Press: Southampton, Boston, pp. 390-398, 2003.

[8] Alleyne, D.N. \& Cawley, P., A two - dimensional Fourier transform method for the measurement of propagating multimode signals. Journ. Acoust. Soc. Am., 89(3), pp. 1159 - 1168, 1991.

[9] Sukatskas, V. \& Volkovas, V., A Technique of Signal Processing for Interferometric Estimation of the Amount of Deposit in Pipes. Russ. Journ. of NDT., 41(7), pp. $430-435,2005$. 